\title{
Olfactory Learning-Induced Long-Lasting Enhancement of Descending and Ascending Synaptic Transmission to the Piriform Cortex
}

\author{
Yaniv Cohen, Iris Reuveni, Edi Barkai, and Mouna Maroun \\ Departments of Biology and Neurobiology, Faculty of Science and Science Education, University of Haifa, Haifa 31905, Israel
}

\begin{abstract}
Learning of a particularly difficult olfactory-discrimination (OD) task results in acquisition of rule learning. This remarkable enhancement in learning capability is accompanied by long-term enhancement of synaptic connectivity between piriform cortex (PC) pyramidal neurons. Because successful performance in the OD task requires integration of information about the identity and also about the reward value of odors, it is likely that a higher-order brain area would also be involved in rule learning acquisition and maintenance. The anterior PC (APC) receives a strong ascending input from the olfactory bulb, carrying information regarding olfactory cues in the environment. It also receives substantial descending input from the orbitofrontal cortex (OFC), which is thought to play an important role in encoding the predictive value of odor stimuli. Using in vivo recordings of evoked field postsynaptic potentials, we characterized the physiological properties of projections to APC from the OFC and examined whether descending and ascending synaptic inputs to the piriform cortex are modified after OD learning. We show that enhanced learning capability is accompanied by long-term enhancement of synaptic transmission in both the descending and ascending inputs. Long-term synaptic enhancement is not accompanied by modifications in paired-pulse facilitation, indicating that such modifications are likely postsynaptic. Predisposition for long-term potentiation induction was affected by previous learning, and surprisingly also by previous exposure to the odors and training apparatus. These data suggest that enhanced connectivity between the APC and its input sources is required for OD rule learning.
\end{abstract}

Key words: olfactory learning; piriform cortex; orbitofrontal cortex; pyramidal neurons; fPSP; synaptic enhancement

\section{Introduction}

Learning of a particularly difficult olfactory-discrimination (OD) training paradigm results in acquisition of rule learning, manifested in a dramatic enhancement of learning capability. OD rule learning is accompanied by a series of profound physiological and morphological modifications in piriform cortex (PC) pyramidal neurons. In particular, intrinsic neuronal excitability is increased (for review, see Saar and Barkai, 2003), synaptic transmission is enhanced (Saar et al., 1999, 2002), and the number of dendritic spines is increased (Knafo et al., 2001). Such OD learning-related modifications are not confined to a subpopulation of cells but are spread throughout the neuronal ensemble (Saar et al., 1999; Knafo et al., 2001).

Because successful performance in the OD task requires integration of information about the identity and about the reward value of odors, it is likely that an additional brain area, of higher order than the olfactory cortex, would also be involved in rulelearning acquisition and maintenance.

The orbitofrontal cortex (OFC) is a main source of descend-

Received Jan. 15, 2008; revised March 27, 2008; accepted April 6, 2008

This work was supported by a grant from the United States-Israel Binational Science Foundation and the Israel Science Foundation (E.B.)

Correspondence should be addressed to Dr. Edi Barkai, Department of Biology, Faculty of Sciences, Haifa University, Haifa 31905, Israel. E-mail: ebarkai@research.haifa.ac.il.

DOI:10.1523/JNEUROSCI.0178-08.2008

Copyright $\odot 2008$ Society for Neuroscience $\quad$ 0270-6474/08/286664-06\$15.00/0 ing input to the PC and is thought to signal the desirability of the expected outcome (Schoenbaum and Roesch, 2005). After learning, OFC neurons encode neutral stimuli that have been associated with motivationally significant stimuli (Rolls, 1996; Schoenbaum et al., 1999; Tremblay and Schultz, 1999, 2000; Roesch and Olson, 2004). The OFC has strong connections with the anterior PC (APC) (Johnson et al., 2000). Therefore, it is a likely candidate to have a central role in the OD task, where choosing the correct odor is rewarded with drinking water.

In particular, the OFC was suggested to use information regarding motivational significance of olfactory cues and to selection and execution of behavioral strategy that would lead to reward acceptance (Schoenbaum et al., 1999). Accordingly, firing activity in the OFC during OD training appears to reflect the value of predicted outcomes, events, or consequence; OFC neurons are affected more by the odor valance than by its identity (Schoenbaum and Eichenbaum, 1995). During odor sampling, the vast majority of cue-selective cells in the OFC neurons developed selective response to the odor cues only after accurate choice performance (Schoenbaum et al., 1999). Damage to the OFC impairs the acquisition and retention of odor discriminations (Eichenbaum et al., 1980, 1983), further strengthening the notion of its central role in OD learning.

The main ascending input to the PC, originating in the olfactory bulb (OB), may also be modified after learning (Roman et al., 1987; Litaudon et al., 1997; Wilson, 2003; Sevelinges et al., 
2004). Such long-lasting enhancement may further increase the excitability of the PC network, thus adding weight to the role of the OFC in controlling and directing the activity of the PC.

The purpose of the present study was to explore whether OD learning is accompanied by long-lasting enhancement in the strength of synaptic connectivity in both the descending and ascending pathways to the PC.

\section{Materials and Methods \\ Animal training}

Age-matched, young adult, Sprague Dawley male rats (5-6 weeks of age) were used. Before training, they were maintained on a $23.5 \mathrm{~h}$ waterdeprivation schedule, with food available ad libitum. OD training protocol was performed with commercial odors that are used regularly in the cosmetics and food industry. Olfactory training consisted of 20 trials per day for each rat as described previously (Saar et al., 1999). Briefly, in each trial, the rat had to choose between two odors (positive and negative cue) presented simultaneously. Rats designated to the trained group were rewarded after choosing the positive cue. The criterion for learning was at least $80 \%$ positive cue choices in the last 10 trials of a training day. Rats in the pseudotrained group were rewarded in a random manner in $50 \%$ of the trials, regardless of the odor they chose. Rats in the naive group were water deprived but not exposed to the maze. Rats were trained to discriminate between two pairs of odors to confirm that rule learning was achieved. For a more detailed description of the behavioral paradigm, see the study by Saar et al. (1999).

As described previously (Saar et al., 1999), rats indeed learned the second pair of odors much faster than the first pair (7-8 d of training for the first pair and $1-2 \mathrm{~d}$ for the second pair). In vivo recordings were performed $3 \mathrm{~d}$ after training completion, when most cellular modifications are present (Saar et al., 1999, 2002; Knafo et al., 2001).

\section{Stimulation and recordings}

Apparatus and surgery. Three days after the training completion, rats were anesthetized (with $40 \%$ urethane, $5 \%$ chloral hydrate in saline, and $0.5 \mathrm{ml} / 100 \mathrm{~g}$, i.p.) and placed in a stereotaxic frame with body temperature maintained at $37 \pm 0.5^{\circ} \mathrm{C}$. The procedure was performed in a strict accordance with the Haifa University regulations and guidelines of the National Institutes of Health.

Small holes were drilled in the skull to allow insertion of electrodes in the brain. A recording microelectrode (glass, tip diameter of 2-5 $\mu \mathrm{m}$, filled with $2 \mathrm{M} \mathrm{NaCl}$, resistance of 1-4 M $\Omega$ ) was slowly lowered into the right APC (3.7 $\mathrm{mm}$ anterior to bregma; $3.3-3.6 \mathrm{~mm}$ lateral; $5.2-5.5 \mathrm{~mm}$ below the pial surface).

For stimulating the descending pathway, a bipolar $125 \mu \mathrm{m}$ stimulating electrode was implanted in the Ventralis lateralis pars oralis of the right OFC, which has strong reciprocal connections with the APC (Illig, 2005). Electrode position was $3.2 \mathrm{~mm}$ anterior to bregma, 2-2.4 mm lateral, and $4-4.5 \mathrm{~mm}$ below the pial surface (Fig. $1 \mathrm{~A}$ ). For stimulating the ascending pathway, a bipolar $125 \mu \mathrm{m}$ stimulating electrode was implanted into the right $\mathrm{OB}(7.9 \mathrm{~mm}$ anterior to bregma; $1.1 \mathrm{~mm}$ lateral; $2 \mathrm{~mm}$ below the surface of the bulb).

Evoked responses were digitized $(10 \mathrm{kHz})$ and analyzed using the Cambridge Electronic Design 1401+ and its Spike 4 software. Off-line measurements were made of the amplitude of field postsynaptic potential (fPSP) using averages of five successive responses to a given stimulation intensity applied at $0.1 \mathrm{~Hz}$. Test stimuli (monopolar pulses, $100 \mu \mathrm{s}$ duration) were delivered at $0.1 \mathrm{~Hz}$. After positioning the electrodes, the rats were left for $30 \mathrm{~min}$ before commencing the experiment.

\section{Protocol for input-output curve}

Input-output (I-O) functions for stimulus intensity versus fPSP magnitude were recorded in response to increasing intensities of stimulation from 0.3 to $10 \mathrm{~mA}$ applied at $0.1 \mathrm{~Hz}$. I-O curves were constructed by using averages of five successive responses to a given stimulation intensity versus the entire range of intensities sampled. Only those experiments having stable baselines and stable I-O curves over multiple trials were included in the analysis.
Paired-pulse facilitation induction

A typical average of five responses to pairs of stimuli applied at $0.1 \mathrm{~Hz}$ with interstimulus intervals (ISIs) of $15 \mathrm{~ms}$ was measured. Paired-pulse facilitation (PPF) was determined by calculating the ratio between the amplitude of the second and first PSPs (PSP2/PSP1).

\section{Long-term potentiation induction}

Long-term potentiation (LTP) was induced by applying theta burst stimulation (TBS) to the OFC or OB (three sets of 10 trains; each train consisted of 10 pulses at $100 \mathrm{~Hz}$; intertrain interval, $200 \mathrm{~ms}$; interest interval, $1 \mathrm{~min}$ ). Field potentials were recorded from the APC for $60 \mathrm{~min}$ after the TBS to the OFC/OB. LTP was measured as an increase in fPSP amplitude. LTP in the descending OFC-APC pathway was applied at stimulus intensity that evoked an fPSP with amplitude of 1-1.2 mV. Stimulus intensity in the ascending pathway was adjusted to evoke an FPSP with amplitude of $40 \%$ of the maximal response.

\section{Histology}

After termination of the electrophysiological recordings, lesions were made by passing anodal currents (10 $\mathrm{mA}$ for $2 \mathrm{~min}$ ) through the stimulating electrode and recording electrode. The brains were removed and frozen with dry ice. Brain slices of $60 \mu \mathrm{m}$ were cut using cryostat. The electrode tract and lesion locations were identifiable under a light microscope.

\section{Statistical analysis}

For all the electrophysiological measurements, between-groups comparison was done using one-way ANOVA for the three groups (naive, trained, and pseudotrained), and post hoc multiple $t$ tests were then applied to compare the groups (trained, pseudotrained, and naive). Student's $t$ test was used to compare the groups.

\section{Results \\ Basic synaptic responses evoked by stimulating the descending and ascending pathways}

We first examined the responses to stimulations applied to the $\mathrm{OFC}$ and $\mathrm{OB}$ at different vertical positions of the recording electrode (Fig. $1 B$ ). Because the responses in the APC to stimulation of the OB are well documented (Ketchum and Haberly, 1993), we used these responses to determine whether the recording electrode is positioned to record synaptic responses in the proximal dendrites of layer II pyramidal neurons (Fig. $1 B$, bottom traces).

Stimulating the descending pathway (OFC-APC) resulted in a typical single-peak fPSP with averaged delay of 7-9 ms, suggesting that the connection is monosynaptic (Fig. 1C, left trace). In the ascending pathway (OB-APC), stimulation induced fPSP in the APC (5.5 mm vertical position) consisting of two synaptic components. These two components were identified previously as an early monosynaptic response evoked by afferent fibers coming via the lateral olfactory tract to layer Ia, and a late disynaptic response at layer $\mathrm{Ib}$, evoked by reactivation of layer II pyramidal neurons (Ketchum and Haberly, 1993). Accordingly, the averaged delay of the first synaptic response was 6-8 ms, and the second synaptic response was $14-18 \mathrm{~ms}$.

\section{Learning is accompanied by enhanced synaptic strength in the descending pathway}

We first examined whether the descending synaptic connections (the OFC-APC pathway) are strengthened after OD learning. Input-output functions for stimulus intensities versus fPSP amplitudes were obtained by recording the responses to a range of increasing intensities of stimulation (from 0.3 to $1 \mathrm{~mA}$ ) in rats taken from the three groups (naive, pseudotrained, and trained). The amplitude of the field potential increased linearly with the increase in stimulus intensity in the three groups. However, the averaged amplitudes in the trained groups were higher than in the 
two control groups (Fig. 2A). Significant differences $(p<0.05)$ between the average responses for each stimulus were found between the trained $(n=12)$ versus naive $(n=16)$ groups. In addition, a significant difference $(p<0.05)$ was revealed for each stimulation between the pseudotrained $(n=12)$ versus the trained group for stimulus intensities in the range of $0.6-1 \mathrm{~mA}$. The average responses of the naive and the pseudotrained groups did not differ in any of the stimulus intensities. These data support the hypothesis that synaptic connectivity between the OFC and the APC is enhanced after learning.

We have shown previously that OD learning is accompanied by reduced PPF in the intrinsic fibers, indicating that synaptic release is enhanced after learning (Saar et al., 1999). To explore whether the enhanced connectivity in the OFC-APC pathway involves a similar mechanism, we compared the values of PPF between the groups. For the three groups, pairs of stimuli separated by short interstimulus intervals of $15 \mathrm{~ms}$ did not result in amplification or depression of the second response in a pair. Accordingly, the mean values of these groups were similar for all groups $(0.97 \pm 0.17$, $n=7$ for naive; $1.084 \pm 0.20, n=8$ for trained; and $1.177 \pm 0.38, n=9$ for pseudotrained), lending support to the notion that learning-induced enhancement in the fPSP amplitude is maintained via a postsynaptic modification.

\section{Learning is accompanied by enhanced synaptic strength in the ascending pathway}

We next examined whether long-lasting synaptic enhancement exists also in ascending pathway (OB-APC). Input-output functions for stimulus intensity versus fPSP amplitudes were recorded in response to increasing intensities of stimulation (from 1 to 10 $\mathrm{mA})$.

The early synaptic response evoked by the afferent connections onto the pyramidal neurons (Schwob et al., 1984; Stripling and Patneau, 1999) was significantly enhanced after learning. Such enhancement was apparent throughout the range of stimulus intensities (1-10 mA). Notably, a significant difference was also found between the pseudotrained and naive groups (Fig. $2 B$ ). The second synaptic response, evoked by local axon interconnecting pyramidal neurons (the intrinsic fibers) within the APC, was also strongly enhanced after learning throughout the range of stimulus intensities (Fig. 2C). In contrast to responses evoked by the afferent connections, here, the responses from pseudotrained versus naive groups were similar.

Predisposition for LTP induction in descending pathways is reduced after exposure to the maze in a nonlearning-specific manner

We have shown previously that the predisposition for LTP induction within the piriform cortex is reduced after learning (Lebel et
B

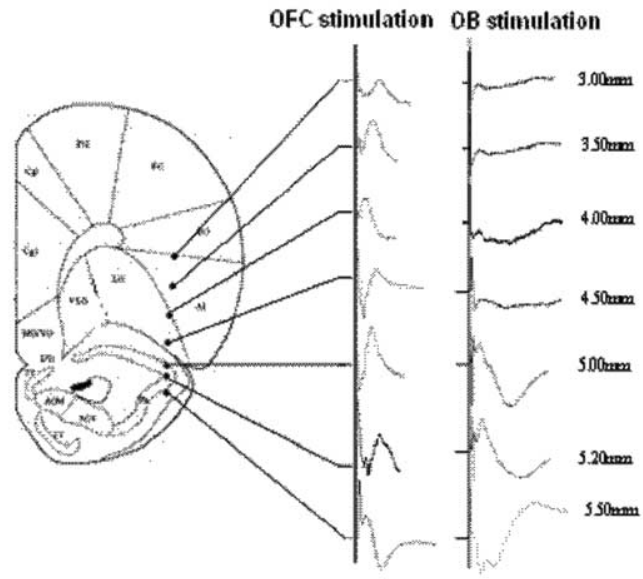

ascending pathway $(O B-P C)$

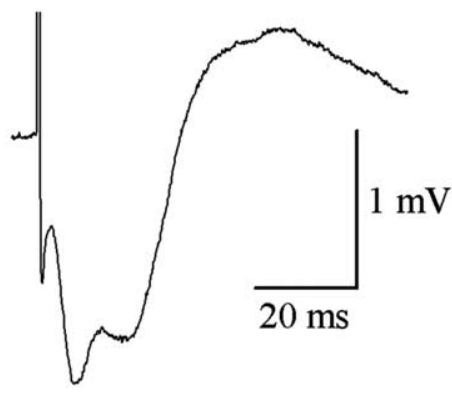

Figure 1. Depth profiles of evoked synaptic responses in the piriform cortex. $A$, Location of stimulating electrode in the OFC. $\boldsymbol{B}$, Depth profiles or synaptic potential recorded in the piriform cortex in response to stimuli applied in the descending (OFC-APC) and ascending (OB-APC) pathways. $\boldsymbol{C}$, Typical responses recorded in layer Ib (B, bottom traces) in response to stimuli applied in the ( is followed by later synapticrespor In the ascending pathway, the first afferent response is followed by a second response, which originates from activation of the association connections between layer II pyramidal neurons.

al., 2001; Quinlan et al., 2004). Here, we examined whether a similar effect occurs also in the ascending and descending pathways.

For LTP induction in the descending pathway, the stimulus intensity was adjusted to evoke an fPSP with amplitude of $\sim 1$ $\mathrm{mV}$. Subsequently, repetitive theta burst stimulation was applied. Such stimulation induced LTP in naive rats $(26.9 \pm 9.9 \% ; n=8)$ but not in trained rats $(2.94 \pm 2.5 \% ; n=7)$ and pseudotrained rats $(7.85+9.3 \% ; n=10)$ (Fig. $3 A)$.

LTP in the OB-APC pathway was much less readily induced, as shown previously (Poo and Isaacson, 2007), for this pathway for young adult rats. Although a very moderate LTP was induced in naive and in pseudotrained rats, no enhancement was induced in trained rats (Fig. $3 B$ ). However, although a tendency toward a difference between the trained and the two control groups was observed, no significant difference was found between groups.

\section{Discussion}

Several anatomical studies point to the existence of direct descending projections from the OFC to the APC (Illig, 2005). Here, we describe the physiological properties of these projections and that they are strengthened after OD learning. Such 

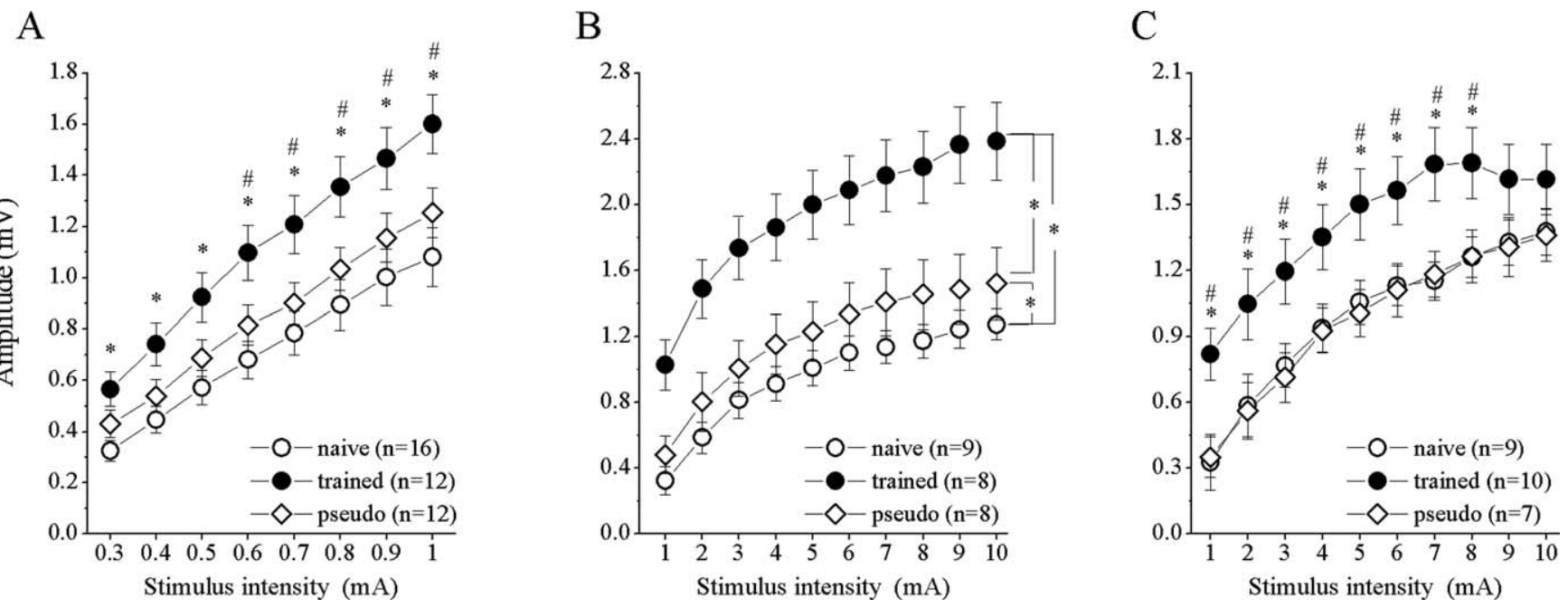

Figure 2. Synaptic connectivity in both pathways is enhanced after learning. $A$, Analysis of the input- output function in OFC-APC pathway. Learning-induced enhanced synaptic responses are evident throughout the range of stimulus intensities. Values represent mean $\pm \mathrm{SE}\left({ }^{\#} p<0.05\right.$, significant difference from pseudotrained groups; ${ }^{*} p<0.05$, significant difference from the naive group). The pseudo-trained and naive groups did not differ significantly at any stimulus intensity. $\boldsymbol{B}$, Analysis of the input- output function of the first synaptic response in the OB-APC pathway. The averaged amplitudes of the first synaptic response, representing activation of the afferent fibers, is significantly higher after learning, compared with the naive and pseudotrained groups throughout the range of stimulus intensities. Also, the averaged amplitudes of the pseudotrained group are significantly higher than the values of the naive group. C, Analysis of the input- output function of the second synaptic response in the OB-APC pathway. The averaged amplitudes of the second synaptic response, representing activation of the intrinsic intracortical fibers is significantly higher after learning, compared with the naive and pseudotrained groups. The two control groups did not differ between them. ${ }^{\#} p<0.05$, significant difference from pseudotrained groups; ${ }^{*} p<0.05$, significant difference from the naive group. The pseudotrained and naive groups did not differ significantly at any of the stimulus intensities. $n$, Number of rats. Values represent mean $\pm \mathrm{SE}$.

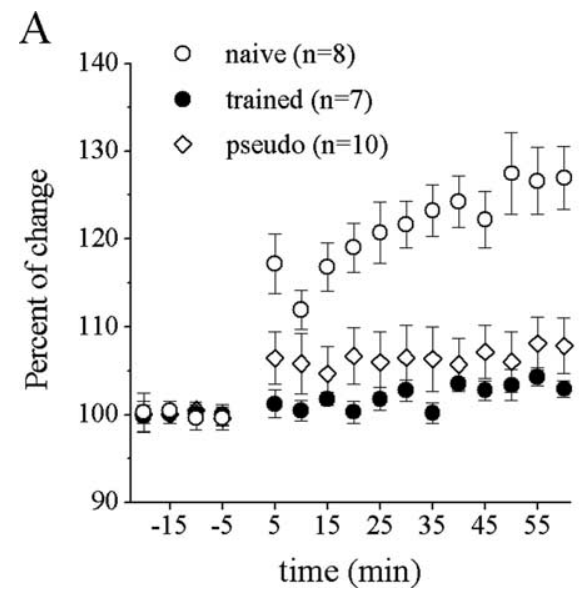

Figure 3. Activity-induced modification in predisposition for LTP induction. $A$, LTP induction in OFC-APC pathway. Although TBS induced LTP in the naive group, no potentiation was observed in the trained and pseudotrained groups. $n$, Number of rats. Values represent mean \pm SE. $B$, In the OB-APC pathway, a mild LTP after TBS is observed in the naive and the pseudotrained groups, whereas no potentiation was recorded in the trained group. $n$, Number of rats. Values represent mean $\pm S E$.

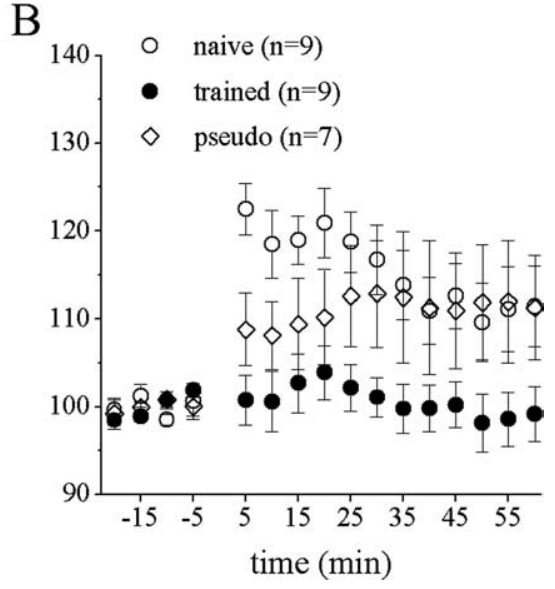

learning-induced enhancement is not restricted to the descending pathway; the connectivity in the ascending OB-APC pathway is also strengthened after learning.

Learning-related enhancements in the descending pathway The short latency of the evoked fPSP in the OFC-APC pathway and its stability suggest that the connection is monosynaptic. Thus, learning-induced modifications in synaptic strength should occur at synaptic connections onto layer II pyramidal neurons. We observed a significant increase in the amplitude of the fPSP in trained rats throughout a wide range of stimulus intensities. Such a uniform difference between groups that persists with increasing stimulus intensity, that presumably increases the number of activated descending fibers, suggests that learninginduced enhancement in synaptic connectivity occurs in most of the synapses activated in this pathway. That pairedpulse facilitation was not modified by learning lends support to the notion that synaptic strengthening is caused by postsynaptic modifications in the piriform cortex pyramidal neurons.

Previous work has shown that OD learning is accompanied by long-lasting postsynaptic modifications in these neurons: the intrinsic excitability of the neurons is enhanced (Saar and Barkai, 2003), the rise time of PSPs evoked by activating the associative fibers interconnecting the pyramidal neurons is reduced (Saar et al., 2002), and the number of synaptic spines is increased (Knafo et al., 2001). These modifications are all present $3 \mathrm{~d}$ after learning and could potentially account for strengthening the synaptic connectivity between the orbitofrontal and the piriform cortices.

\section{Learning-induced modulation of LTP induction in the descending pathway}

Previous research showed that the predisposition for LTP induction in the piriform cortex is reduced after OD learning (Lebel et al., 2001; Quinlan et al., 2004). Such learninginduced reduction in the ability to induce LTP does not necessarily reflect the difficulty in further strengthening synaptic connections that were enhanced during the learning process. Rather, it may be a consequence of a different modification, for example, such that was shown in the subunit composition of the NMDA receptor in piriform cortex neurons (Quinlan et 
al., 2004). Suppression of LTP induction may function as a protective mechanism that prevents overstrengthening of undesirable connections.

That LTP could not be induced in pseudotrained rats, although the fPSP was not enhanced significantly in these animals, suggests that such a mechanism may be present and related to exposure to the training apparatus and procedure. Together with the finding that the synaptic connections in ascending pathway are also mildly enhanced after learning, these data suggest that not all changes should be attributed to the learning process per se. However, most of the long-lasting modulation was observed in trained rats only, implying that in general, synaptic enhancement is related specifically to rule learning.

\section{Learning-related enhancements in the ascending pathway}

Analysis of the input-output functions of synaptic responses evoked by stimulating the ascending (OB-APC) pathway revealed a significant learning-induced synaptic strengthening enhancement in the responses of the trained rats after the OD task. Such enhancement was much similar to that observed for the descending pathway, with one notable exception: the first synaptic response is enhanced not only in trained rats but also in pseudotrained rats, although to a much lesser extent. Therefore, the ascending synaptic connections are enhanced by exposure to the odors or the training apparatus and not only by learning (although learning still accounts for most of the enhancement observed in trained rats). The second synaptic response, reflecting activity of the association fibers interconnecting the pyramidal neurons, is enhanced only after learning, as shown previously in brain slices (Saar et al., 1999, 2002; Brosh et al., 2007). Only moderate LTP was observed after stimulating this pathway. Such a result is consistent with previous observations that LTP induction in this pathway declines rapidly with development (Poo and Isaacson, 2007). However, even such moderate LTP could not be induced after learning, indicating that also, here, learning affects subsequent activity-dependent synaptic plasticity.

\section{Functional implications of enhanced connectivity}

Our study suggests that the OFC may play a role in modulating response properties of neurons in piriform cortex to olfactory information, which is conveyed from the OB. The OFC-APC projections terminate on the apical dendrites of the pyramidal neurons in layer $\mathrm{Ib}$ and thus in proximity to the afferent input on the apical dendritic tree of pyramidal cells in ventral APC (Haberly, 1998; Illig, 2005). Our results suggest that, after OD learning, the capability of the OFC to modulate afferent fiberinduced activity in piriform cortex, is greatly enhanced. Descending modulation from a high brain area may act to ensure that cells responsive to a particular odor fire in an appropriate behavioral context. Such modulation is particularly significant in light of the finding that cells in piriform cortex can display robust responses to nonolfactory cues during a behavioral task (Schoenbaum and Eichenbaum, 1995). It would also modulate the response of the PC pyramidal neurons to the ascending input for the olfactory bulb and the intracortical inputs, both of which are enhanced by learning.

That the projections from OFC to piriform cortex terminate in the association fiber layers within APC (Illig, 2005) also raises the possibility that the OFC may play a role in initiating activity within piriform cortex in the absence of an odor stimulus during recall of multimodal memories. In particular, the heavy projection onto the proximal apical dendritic tree of cells in piriform cortex (i.e., layer Ib) from OFC could serve to mimic the effect of afferent fibers during memory retrieval. Subsequent activation of cells in piriform cortex may then allow recall of odors or odorrelated associations. Evidence for such memory-related activity in the piriform cortex has been found in animals (Shimshek et al., 2005) and humans (Li et al., 2006).

We suggest that through the OFC-APC connections, OFC might actively modulate afferent input to APC so that cells fire in certain contexts but not others, and that the strength of these connections is greatly enhanced after OD learning, for periods of days at least. Moreover, OFC input could potentially initiate activity in the APC in the absence of any odor stimulation, allowing for recall of odors and odor-related associations. The overall strengthening of the descending pathway suggests that the specificity of the evoked odor memory is achieved not by these inputs but by specific synaptic connections that were strengthened within the piriform cortex network, during the learning process.

\section{References}

Brosh I, Rosenblum K, Barkai E (2007) Learning-induced modulation of SK channels-mediated effect on synaptic transmission. Eur J Neurosci 26:3253-3260.

Eichenbaum H, Shedlack KJ, Eckmann KW (1980) Thalamocortical mechanisms in odor-guided behavior. I. Effects of lesions of the mediodorsal thalamic nucleus and frontal cortex on olfactory discrimination in the rat. Brain Behav Evol 17:255-275.

Eichenbaum H, Morton TH, Potter H, Corkin S (1983) Selective olfactory deficits in case H.M. Brain 106:459-472.

Haberly LB (1998) Olfactory cortex. In: The synaptic organization of the brain (Shepherd GM, ed), pp 377-416. New York: Oxford UP.

Illig KR (2005) Projections from orbitofrontal cortex to anterior piriform cortex in the rat suggest a role in olfactory information processing. J Comp Neurol 488:224-231.

Johnson DM, Illig KR, Behan M, Haberly LB (2000) New features of connectivity in piriform cortex visualized by intracellular injection of pyramidal cells suggest that "primary" olfactory cortex functions like "association" cortex in other sensory systems. J Neurosci 20:6974-6982.

Ketchum KL, Haberly LB (1993) Membrane currents evoked by afferent fiber stimulation in rat piriform cortex. I. Current source-density analysis. J Neurophysiol 69:248-260.

Knafo S, Grossman Y, Barkai E, Benshalom G (2001) Olfactory learning is associated with increased spine density along apical dendrites of pyramidal neurons in the rat piriform cortex. Eur J Neurosci 13:633-638.

Lebel D, Grossman Y, Barkai E (2001) Olfactory learning modifies predisposition for long-term potentiation and long-term depression induction in the rat piriform (olfactory) cortex. Cereb Cortex 11:485-489.

Li W, Luxenberg E, Parrish T, Gottfried JA (2006) Learning to smell the roses: experience-dependent neural plasticity in human piriform and orbitofrontal cortices. Neuron 52:1097-1108.

Litaudon P, Mouly AM, Sullivan R, Gervais R, Cattarelli M (1997) Learning-induced changes in rat piriform cortex activity mapped using multisite recording with voltage sensitive dye. Eur J Neurosci 9:1593-1602.

Poo C, Isaacson JS (2007) An early critical period for long-term plasticity and structural modification of sensory synapses in olfactory cortex. J Neurosci 27:7553-7558.

Quinlan EM, Lebel D, Brosh I, Barkai E (2004) A molecular mechanism for stabilization of learning-induced synaptic modifications. Neuron. 41:185-192.

Roesch MR, Olson CR (2004) Neuronal activity related to reward value and motivation in primate frontal cortex. Science 304:307-310.

Rolls ET (1996) The orbitofrontal cortex. Philos Trans R Soc Lond B Biol Sci 351:1433-1443; discussion 1443-1444.

Roman F, Staubli U, Lynch G (1987) Evidence for synaptic potentiation in a cortical network during learning. Brain Res 418:221-226.

Saar D, Barkai E (2003) Long-term modifications in intrinsic neuronal properties and rule learning in rats. Eur J Neurosci 17:2727-2734.

Saar D, Grossman Y, Barkai E (1999) Reduced synaptic facilitation between 
pyramidal neurons in the piriform cortex after odor learning. J Neurosci 19:8616-8622.

Saar D, Grossman Y, Barkai E (2002) Learning-induced enhancement of postsynaptic potentials in pyramidal neurons. J Neurophysiol 87:2358-2363.

Schoenbaum G, Eichenbaum H (1995) Information coding in the rodent prefrontal cortex. I. Single-neuron activity in orbitofrontal cortex compared with that in pyriform cortex. J Neurophysiol 74:733-750.

Schoenbaum G, Roesch M (2005) Orbitofrontal cortex, associative learning, and expectancies. Neuron 47:633-636.

Schoenbaum G, Chiba AA, Gallagher M (1999) Neural encoding in orbitofrontal cortex and basolateral amygdala during olfactory discrimination learning. J Neurosci 19:1876-1884.

Schwob JE, Haberly LB, Price JL (1984) The development of physiological responses of the piriform cortex in rats to stimulation of the lateral olfactory tract. J Comp Neurol 223:223-237.

Sevelinges Y, Gervais R, Messaoudi B, Granjon L, Mouly AM (2004) Olfac- tory fear conditioning induces field potential potentiation in rat olfactory cortex and amygdala. Learn Mem 11:761-769.

Shimshek DR, Bus T, Kim J, Mihaljevic A, Mack V, Seeburg PH, Sprengel R, Schaefer AT (2005) Enhanced odor discrimination and impaired olfactory memory by spatially controlled switch of AMPA receptors. PLoS Biol 3:2017-2030.

Stripling JS, Patneau DK (1999) Potentiation of late components in olfactory bulb and piriform cortex requires activation of cortical association fibers. Brain Res 841:27-42.

Tremblay L, Schultz W (1999) Relative reward preference in primate orbitofrontal cortex. Nature 398:704-708.

Tremblay L, Schultz W (2000) Reward-related neuronal activity during gonogo task performance in primate orbitofrontal cortex. J Neurophysiol 83:1864-1876.

Wilson DA (2003) Rapid, experience-induced enhancement in odorant discrimination by anterior piriform cortex neurons. J Neurophysiol 90:6572 . 